\title{
Percepciones campesinas sobre los cambios climáticos en Huamuchapa, Guerrero
}

Peasant perceptions about climate change in Huamuchapa, Guerrero

Percepções dos camponeses sobre as mudanças climáticas em Huamuchapa, Guerrero

Josefina Munguia-Aldama

Universidad Autónoma de Guerrero, México josefinamunguia@hotmail.com https://orcid.org/0000-0003-0527-2884

Eleuterio Campos-Hernández

Universidad Autónoma de Guerrero, México ecamposh@hotmail.com https://orcid.org/0000-0002-4117-0271

Rosalba Díaz-Vásquez

Universidad Autónoma de Guerrero, México rosaldiaz@yahoo.com.mx https://orcid.org/0000-0001-5304-1840

Mario Martínez-Rescalvo Universidad Autónoma de Guerrero, México rescalvo@yahoo.com https://orcid.org/0000-0002-5842-0740

Roxana Reyes-Ríos Universidad Autónoma de Guerrero, México roxx_r@hotmail.com https://orcid.org/0000-0003-4376-4687 


\section{Revista lberoamericana de las Ciencias Sociales y Humanísticas}

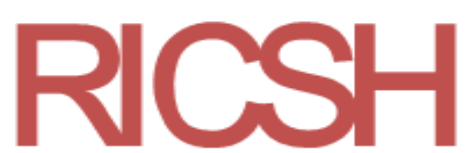

ISSN: $2395-7972$

\section{Resumen}

En el presente trabajo se analiza la perspectiva que tienen campesinos de la comunidad de Huamuchapa, municipio de Tecoanapa, Guerrero, México, sobre los cambios del clima (su origen e implicaciones), así como la percepción de indicadores bioclimáticos a nivel local. Mediante una metodología mixta, se realizó un análisis estadístico descriptivo. La información fue recabada mediante observación directa, entrevistas semiestructuradas, recorridos en parcelas y una encuesta sociodemográfica y de producción campesina aplicada a $30 \%$ de las familias de la localidad. Se encontró que estos campesinos tenían un conocimiento incipiente del cambio climático en términos conceptuales, lo que no impide que perciban claramente que el clima ha venido cambiando. Sus explicaciones sobre dicho fenómeno giran en torno a la naturalidad de este, desconocimiento, creencias y actividades antropogénicas. Los efectos del cambio climático son reconocidos en las modificaciones de la temperatura y la precipitación, variables que impactan directamente en el cultivo del maíz y en la falta de disponibilidad de agua para uso doméstico; además, los campesinos reconocen que los elementos que se han modificado son relevantes para la sobrevivencia de la comunidad, la familia y las próximas generaciones. En conclusión, los campesinos viven los cambios del clima, los identifican y los explican de acuerdo con sus experiencias y creencias. De hecho, tienen gran disposición para participar en acciones de adaptación y son conscientes de las consecuencias de sus actos al entorno que los rodea. Frente a las adversidades climáticas prueban y adoptan prácticas resolutivas para disponer de maíz.

Palabras clave: adaptación climática, cambios climáticos, percepción climática campesina.

\section{Abstract}

This research analyzes the peasant perspective about climate change by peasant from the community of Huamuchapa, municipality of Tecoanapa, in Guerrero, Mexico, its origin and implications, and the perception of bioclimatic indicators at the local level. We used a mixed methodology, a descriptive statistical analysis was carried out, the information derived from direct observation, semi-structured interviews, tours of plots and a socio-demographic and peasant production survey applied to $30 \%$ of the families in the locality. It was found that these peasants had an incipient knowledge of climate change in conceptual terms, which does 


\section{Revista Iberoamericana de las Ciencias Sociales y Humanísticas}

ISSN: $2395-7972$

not prevent them from clearly perceiving that the climate has been changing and their explanations about it revolve around its naturalness, ignorance, their beliefs and activities anthropogenic. The effects of climate change are recognized in the changes in temperature and precipitation, which have a direct impact on the cultivation of maize, and the lack of availability of water for domestic use, in addition, farmers recognize and reflect that the elements that are they have modified are relevant for the survival of the community, the family and the next generations. In conclusion, peasants experience climate changes, identify and explain them according to their experiences and beliefs, are very willing to participate in adaptation actions, they are aware of the effects considered "damage" to the environment that surrounds them and contribution of their own acts to them. Faced with climatic adversities, they try and adopt decisive practices to dispose of maize.

Keywords: climate adaptation, climate change, perception peasant climate.

\section{Resumo}

Este artigo analisa a perspectiva que os camponeses da comunidade de Huamuchapa, município de Tecoanapa, Guerrero, México, têm sobre as mudanças climáticas (suas origens e implicações), bem como a percepção dos indicadores bioclimáticos em nível local. Utilizando uma metodologia mista, foi realizada uma análise estatística descritiva. As informações foram coletadas por meio de observação direta, entrevistas semiestruturadas, passeios em parcelas e levantamento sociodemográfico e da produção camponesa aplicado a $30 \%$ das famílias da localidade. Constatou-se que esses agricultores possuíam um conhecimento incipiente sobre as mudanças climáticas em termos conceituais, o que não os impede de perceber claramente que o clima está mudando. Suas explicações sobre esse fenômeno giram em torno de sua naturalidade, ignorância, crenças e atividades antropogênicas. Os efeitos das mudanças climáticas são reconhecidos nas mudanças de temperatura e precipitação, variáveis que impactam diretamente no cultivo do milho e na falta de disponibilidade de água para uso doméstico; Além disso, os camponeses reconhecem que os elementos modificados são relevantes para a sobrevivência da comunidade, da família e das próximas gerações. Concluindo, os camponeses vivem as mudanças do clima, identificam-nas e explicam-nas de acordo com as suas experiências e crenças. Na verdade, eles estão muito dispostos a participar de ações de adaptação e estão cientes das 


\section{Revista Iberoamericana \\ de las Ciencias Sociales y Humanísticas}

ISSN: $2395-7972$

consequências de suas ações para o ambiente ao seu redor. Diante das adversidades climáticas, eles tentam adotar práticas decisivas para o descarte do milho.

Palavras-chave: adaptação ao clima, mudanças climáticas, percepção do clima camponês. Fecha Recepción: Marzo 2020

Fecha Aceptación: Enero 2021

\section{Introducción}

El cambio climático es, actualmente, uno de los problemas globales de mayor relevancia debido a los fenómenos ambientales que genera, los cuales impactan la biodiversidad, los ecosistemas, los procesos productivos, la infraestructura y la salud (VélezTorres, Santos-Ocampo, De la Tejera-Hernández y Monterroso-Rivas, 2016). De hecho, la Organización Mundial de la Salud (OMS) ha señalado que el ambiente es el responsable de una cuarta parte de las muertes en el planeta, ya que provoca nuevas enfermedades que se han cuadruplicado en los últimos 50 años debido a la perturbación de los ecosistemas (Edwards, García y Walkins, 25 de marzo de 2020).

En el caso concreto de México, sus características geográficas, climáticas, orográficas e hidrológicas contribuyen a que sea una de las zonas más vulnerables del mundo por el cambio climático (González, Silva, Ávila, Moncayo-Estrada, Cruz y Ceja, 2017). Por ejemplo los huracanes Ingrid y Manuel en Guerrero provocaron desastres en las regiones Costa Grande y Costa Chica con costos en varios millones de dólares (Detta, 2014).

Expertos han demostrado con suficiencia que el clima está cambiando a un ritmo sin precedente por las actividades antropogénicas. Tales afirmaciones se basan en el análisis de indicadores $^{1}$ a nivel global, los cuales prevén el incremento en la temperatura, los cambios en los patrones de precipitación, entre otros. A los cuales se agregan los de tipo cualitativo, en el sentido de lo expresado por la Agencia Europea del Medio Ambiente (AEMA, 2006).

Si bien es cierto que desde los gobiernos los esfuerzos para mitigar y adaptarse al cambio climático han ido en aumento, aún falta mucho por hacer. En este sentido, para los países de América Latina, la Comisión Económica para América Latina y el Caribe (Cepal)

\footnotetext{
${ }^{1}$ Un indicador es, comúnmente, la síntesis de un conjunto de datos cuantitativos que sirven para representar un fenómeno complejo, al mismo tiempo en términos cualitativos es "un indicio o señal con un grado razonable de certidumbre" (AEMA, 2006, p. 7), que proporciona evidencias de un evento o un comportamiento.
} 


\section{Revista Iberoamericana de las Ciencias Sociales y Humanísticas}

ISSN: $2395-7972$

sostiene que la mitigación y adaptación ${ }^{2}$ son inseparables porque de continuar con emisiones de gases de efecto invernadero al ritmo actual no solo seguirá aumentando la temperatura, sino que tendrá otros efectos. Así, la adaptación implica diseñar medidas que respondan a la naturaleza del cambio tomando en cuenta las condiciones de las distintas poblaciones para ampliar la posibilidad de mejorar la calidad de vida en el medio rural (Bárcena, Samaniego, Peres y Alatorre, 2020).

Ahora bien, percibir el cambio climático como amenaza puede variar entre las poblaciones. La percepción de riesgos depende de conocimientos, actitudes, creencias, juicios y sentimientos atravesados por la experiencia cotidiana, así como por la disposición a actuar en función de la propia valoración de la vida, la salud y los bienes (Urbina, 2015). Las percepciones poblacionales se basan en rasgos, características o indicadores descriptibles no necesariamente medibles que los detallan con suficiencia.

Acercarse a estos entendimientos es útil porque el clima y sus manifestaciones son el resultado de cómo cada individuo percibe, interpreta y se apropia de los eventos; por tanto, los conceptos de clima son construcciones humanas culturales, espaciales e históricas (Mariño, 2011; Soares, García y Manzano, 2018) muchas veces compartidas. Esto significa que cada cultura tiene sus propias concepciones y explicaciones en torno al clima.

Por ejemplo, entre las poblaciones indígenas y campesinas de México existía un conocimiento profundo del clima que les permitía su predicción a través del método conocido como cabañuelas, de aplicación concreta en la agricultura. En ciertos grupos, los conocimientos específicos sobre el clima se perciben y prevén desde la experiencia a través de indicadores bioclimáticos (Ulloa, 2011).

En la actualidad no solo las conceptualizaciones siguen siendo necesarias de conocer, sino también las acciones que realizan los campesinos para enfrentar los efectos climáticos, al convertirse en medidas adaptativas adecuadas a sus necesidades, en ocasiones muy particulares, que están siendo recapacitadas por las posturas científicas. Esto demanda entender a la adaptación como las acciones que toman los ciudadanos para reducir el peso de los efectos adversos (Salazar-Ceballos, Freyle, Tamara y Álvarez-Miño, 2016) y como un conjunto de medidas que se implementan desde abajo. La consideración es que si lo que la

\footnotetext{
${ }^{2}$ Se entiende por mitigación a las acciones para reducir la emisión de gases y compuestos de efecto invernadero a la atmósfera. En cambio, la adaptación se refiere a las "iniciativas y medidas encaminadas a reducir la vulnerabilidad de los sistemas naturales y humanos ante los efectos reales o esperados de un cambio climático" (Instituto Nacional de Ecología y Cambio Climático [INECC], 2018, párr.1).
} 


\section{Revista Iberoamericana de las Ciencias Sociales y Humanísticas}

ISSN: $2395-7972$

gente hace funciona, es válido de ser escuchado y compartido, porque — como señalan Bárcena et al. (2020)— "la inacción también tiene costos" (p. 19).

Las investigaciones en la última década sobre los diversos niveles de conocimiento y percepción del cambio climático en todo el mundo también han aumentado, aunque el reto sigue siendo bajar la escala espacial (Ferrero, Hernández y Zafra, 2014). Los estudios sobre saberes, prácticas y creencias contribuyen a llenar vacíos de información para el diseño de las políticas públicas, en las cuales deben converger los referentes de grupos nacionales, regionales y locales (Corona, 2018) hacia la búsqueda de soluciones integrales cuando sean pertinentes o hacia la definición de estrategias particulares de acuerdo con las condiciones reales. En otras palabras, disponer de información de estudios locales proporciona ciertas ventajas, pues resulta políticamente más fácil proponer, implementar y valorar acciones cuando hay acuerdo global (Estrada, 2017).

En esta dirección está enfocada la investigación de González et al. (2017), quienes abordan la percepción o conocimiento que tiene sobre el cambio climático la población indígena purépecha del municipio de Chilchota en Michoacán. Los resultados de investigación muestran que la población encuestada reconoce cambios negativos del cambio climático en la salud, las actividades económicas y el bienestar a mediano y largo plazo.

Por otra parte, el estudio en la comunidad de Ahuihuiyuco del estado de Guerrero refleja cómo los campesinos, basados en sus observaciones sobre la relación clima y cultivo de maíz, enfrentan las contingencias modificando sus acciones y las convierten en estrategias adaptativas para atenuar el riesgo agrícola (Munguia-Aldama, Sánchez-Plata, Vizcarra-Bordi y Rivas-Guevara, 2015).

Varios estudios han comprobado que los cambios en la precipitación e incremento en la temperatura son los indicadores más reconocidos, aunque su presencia se asume desde distintas temporalidades, algunos, por cierto, muy recientes (Barrasa, 2017; Ferrero et al., 2014; González et al., 2017; Novión y Estrada, 2011; Pinilla, Rueda, Pinzón y Sánchez, 2012; Vélez-Torres et al., 2016). Mención especial merece la situación del agua y del aire, puesto que la gente común los evalúa como de mayor riesgo (Urbina, 2015).

Existen otras investigaciones que analizan la percepción en habitantes urbanos, cuyos resultados evidencian que aunque las personas conciben al cambio climático como riesgo para la vida, lo ven relativamente distante de sí mismos; es decir, creen que sus efectos ya han comenzado, pero en otros países o lugares, porque no se conecta directamente con su 


\section{Revista Iberoamericana}

de las Ciencias Sociales y

Humanísticas

ISSN: $2395-7972$

trabajo, sus relaciones interpersonales o sus posesiones materiales (Martínez, 2015; Novión y Estrada, 2011; Urbina, 2015).

Con base en estos referentes, el propósito del presente estudio fue analizar la perspectiva que tienen campesinos de la comunidad de Huamuchapa sobre los cambios de clima, origen, implicaciones, así como la percepción de indicadores bioclimáticos a nivel local.

\section{Materiales y métodos}

La investigación se realizó en el poblado de Huamuchapa, municipio de Tecoanapa, en la región de la Costa Chica del estado de Guerrero, México. Esta localidad se ubica entre las siguientes coordenadas: longitud $99^{\circ} 19^{\prime} 03^{\prime}$ ', latitud $16^{\circ} 56^{\prime} 39^{\prime \prime}$ y a una altitud de 720 metros sobre el nivel del mar.

El alcance es descriptivo porque "interpreta lo que es" (Tamayo y Tamayo, 2002), detalla situaciones y acontecimientos. Se aborda el problema usando de forma complementaria procedimientos de enfoque cuantitativo y cualitativo, pues se considera del pluralismo metodológico su carácter integrador (Ametrano, Vestfrid, Bonaparte, Adaro y Huarte, 2017). Se utilizó un modelo en dos etapas (Hernández, Fernández, y Baptista, 2002): en la primera lo cuantitativo y en la segunda lo cualitativo, aunque este último es predominante.

El acceso a la comunidad se logró mediante un acuerdo con el comisario luego de una presentación a población abierta sobre residuos sólidos. La notificación de nuestra presencia quedó a cargo de su persona.

La estrategia cuantitativa fue la encuesta y se denominó encuesta socioeconómica y de producción de maíz, aplicada a una muestra de $30 \%$ de jefes o jefas de familias existentes en la localidad (168 en total).

Por heterogeneidad en la concentración de las viviendas por manzana o área de levantamiento, y por la desigualdad en su tamaño, las manzanas del levantamiento se eligieron al azar a partir de su numeración en mapa. En ellas, mediante técnica de barrido, se encuestó a quienes se encontraban en sus viviendas durante el recorrido. La información fue proporcionada voluntariamente por el jefe o jefa de familia, una vez explicitado el propósito de la encuesta y previa garantía de confidencialidad. 


\section{Revista Iberoamericana de las Ciencias Sociales y Humanísticas}

ISSN: 2395 - 7972

El instrumento de recolección fue un cuestionario estandarizado de 8 secciones y 149 variables. La segunda sección recogió información sobre las características del encuestado. El apartado específico sobre cambio climático que aquí se aborda incluyó 22 reactivos relacionados con indicadores bioclimáticos tales como cambios en la temperatura, cambios en la precipitación, eventos extremos, cambios en la vegetación y riesgos para la colectividad, los cuales se indagaron mediante escala de valoración ordinal asignando un número de menor a mayor intensidad. Los datos reflejan la percepción del jefe o jefa de familia.

Previo a la aplicación se realizó una prueba piloto en otra localidad para validar la calidad del instrumento. La versión final fue aplicada por estudiantes de las licenciaturas en Desarrollo Regional, Sociología y Biología Experimental de la Universidad Autónoma de Guerrero. Aunque los datos se procesaron en el programa SPSS, versión 22, solo se presentan mediante estadísticas descriptivas porque se trata de un primer acercamiento desde esta visión a dicha realidad.

En la estrategia cualitativa se utilizaron la entrevista semiestructurada (Flick, 2007) a informantes clave y la observación directa en parcelas y viviendas. La entrevista recupera de voz de los participantes (lo que saben, piensan y creen en sus propias palabras) (Taylor y Bogdan, 2013). Estas se llevaron a cabo con siete campesinos jefes de familia que cultivan maíz, todos varones, porque es poco usual que las mujeres se hagan cargo de estas labores. Fueron elegidos mediante bola de nieve (Cea, 2001) con apoyo de instrumentos tecnológicos en lugares como su casa, la parcela o la cancha techada de la localidad. El asunto central de las conversaciones fue el cultivo de maíz, mientras que sus posibles daños vinculados a los cambios de clima fueron solo una parte en la diversidad de lo expresado. Las conversaciones tuvieron una duración de una a dos horas continuas o en dos sesiones.

La participación de los informantes clave también fue voluntaria. Una vez se les explicó las intenciones del estudio, se garantizó la confidencialidad y el uso exclusivamente académico de sus informaciones. De hecho, aunque los citamos en los resultados, no es posible identificarlos debido a lo común de sus apellidos entre los habitantes.

La observación directa se realizó sin apoyo de una guía estructurada, mediante recorridos en parcelas en la época de siembra, dispersión de abono y cosecha, así como en las viviendas durante la cosecha, desgrane y almacenamiento de los productos agrícolas. Durante los recorridos se contó con el acompañamiento de una estudiante universitaria para reducir la posibilidad de obstrucción por parte del grupo de autodefensa. 


\section{Revista Iberoamericana}

de las Ciencias Sociales y

Humanísticas

ISSN: $2395-7972$

Los audios y las notas de campo se trascribieron en Word. La información cualitativa se ordenó y clasificó mediante códigos y categorías temáticas. De estas se extrajeron palabras, frases o párrafos significativos que luego fueron articulados en una construcción interpretativa con lo cuantitativo.

\section{Resultados}

Presentamos los hallazgos en dos partes: la primera expone las concepciones de cambio climático y la segunda la percepción de indicadores bioclimáticos, sus afectaciones o consecuencias en los últimos años y perspectivas futuras. Sin embargo, antes de entrar en detalle, describimos algunas de las características del jefe o jefa y de sus familias. La jefatura es predominantemente masculina, aunque $29 \%$ corresponde a mujeres, madres solteras, viudas o abandonadas por la pareja. Además, $94 \%$ de los encuestados son católicos y la escolaridad promedio es de seis años (es decir, con primaria completa y $21 \%$ sin escolaridad). Habitan en casas pequeñas, $45 \%$ de las cuales disponen de un solo cuarto donde cohabita una familia de cinco miembros. Hubo casos con grupos extensos de nueve integrantes conformados por abuelos, suegros, tíos, padres hijos y nietos.

El alimento esencial es el maíz: 96 \% lo cultiva, y consumen en promedio cuatro litros por semana solo para tortilla. El litro ha sido una medida convencional entre los habitantes de la entidad para productos diversos. Algunos entrevistados reportaron consumos de hasta 10 litros de maíz semanalmente, independiente del utilizado para pozole, tamales y otros alimentos.

Un asunto relevante en relación con el tema del cambio climático es el uso de leña para cocinar por todas las familias $(100 \%)$ de Huamuchapa, lo que se refleja en la tala de árboles en la zona para satisfacer dicha demanda.

\section{El concepto del cambio de clima, su origen e implicaciones}

Aunque algunos campesinos han escuchado hablar del cambio climático, aún no disponen de una elaboración conceptual con contenido y significado propio (en este estudio no pudimos recuperarlo), seguramente porque para ellos se trata de un tema de muy reciente discusión y de connotación específica. Aun así, la referencia al clima es una constante en sus vidas, en distintos sentidos y desde hace mucho tiempo. De hecho, es común escuchar que 


\section{Revista Iberoamericana de las Ciencias Sociales y Humanísticas}

ISSN: $2395-7972$

clima y tiempo se usan indistintamente como sinónimos en expresiones como "el tiempo está cambiando mucho" o "ahora el tiempo no es como antes" (Barrera, comunicación personal, 10 de diciembre 2018). Asimismo, suelen otorgar varias connotaciones a la palabra tiempo: “tiempo de secas", "tiempo de calor", "tiempo de lluvias", "tiempo de sequía", "tiempo de frío” (García, comunicación personal, 20 de julio 2019).

Los cambios de clima son asumidos como ciertos por $86 \%$ de los habitantes encuestados, lo cual es más claramente identificado por los hombres que por las mujeres. En este sentido, $60 \%$ de los hombres aceptan la existencia de cambios notorios en el clima en su localidad, porcentaje que se reduce a poco menos de la mitad (26\%) en el caso de las mujeres.

Caso contrario sucede con quienes afirman que no hay cambios en el clima: aquí el porcentaje más alto es para los hombres $(11 \%)$ y apenas $3 \%$ para las mujeres. La siguiente opinión resume esta percepción de estabilidad climática: "El clima es como siempre, desde que me acuerdo es así’” (Roque, comunicación personal, 10 de agosto 2019).

Por otra parte, las razones más comunes para justificar el cambio climático se pueden agrupar en cinco categorías: para $33 \%$ de las personas el origen se encuentra en la tala de árboles por los propios pobladores de la región y 26 \% lo atribuye a la contaminación y a la quema de basura. Asimismo, las creencias ocupan el tercer lugar con $16 \%$ : entre estas, las religiosas registran $5.5 \%$ con expresiones como las siguientes: "Es un castigo de Dios por el mal comportamiento humano" (en especial de los hijos) o porque "se acerca el fin del mundo": "Los hijos maltratan o golpean a los padres" o en cualquier lugar "te levantan, tortura, asesinan o roban, antes no" (Ayodoro, comunicación personal, 31 de octubre 2018). Igualmente, $10.5 \%$ sostiene que "el sol es más caliente porque está descendiendo". La declaratoria de desconocimiento también es relevante, aunque representa $13 \%$. Estos dos tipos de pensamientos (creencias y desconocimiento) alcanzan $29 \%$, lo que refleja la falta de claridad de lo que ocurre tanto en la comunidad como a nivel global.

Por otra parte, $10 \%$ afirma que los cambios de clima son naturales ("así ha sido siempre"), y una pequeña porción (2 \%) menciona específicamente que se debe al calentamiento global.

Los datos, en definitiva, muestran que las explicaciones de $60 \%$ de los campesinos están estrictamente ligadas a actividades antropogénicas: la tala, la contaminación, la quema de basura y el calentamiento global. 


\section{Revista Iberoamericana}

de las Ciencias Sociales y

Humanísticas

ISSN: $2395-7972$

Sabemos que las cifras que se usan para referirse al cambio climático han sido recabadas durante periodos prolongados (por lo menos 30 años); sin embargo, en estas sociedades las percepciones sobre las variaciones climáticas son más recientes (principalmente desde hace unos cinco años, pues solo algunos tienen esta percepción desde hace10, 15 o 30 años).

$\mathrm{Al}$ indagar sobre lo que representan estos cambios en su vida (con valoraciones que van de poco a considerable), $96 \%$ asume que los daños mayores vendrán para las generaciones futuras; no obstante, $93 \%$ estima que ya hay efectos negativos en la comunidad, y cuando se trata de los más cercanos (como la familia) alcanza $97 \%$. Las consecuencias declaradas como más sentidas son la falta de agua, la disminución de las cosechas y la presencia de plagas. De hecho, llama la atención la relación entre los cambios de clima y la presencia de nuevas enfermedades en los humanos (opinión de $16 \%$ de los entrevistados).

\section{Reconocimiento de indicadores bioclimáticos a nivel local}

El reconocimiento de los campesinos de Huamuchapa sobre las sensaciones térmicas distintas, al igual que sus consideraciones sobre las pérdidas que los cambios de clima provocan en los cultivos en cada ciclo agrícola, se perciben mediante indicadores bioclimáticos, referidos constantemente entre un antes y un después. En tal sentido, $56 \%$ siente que la temperatura ha aumentado fuertemente, $38 \%$ concibe incrementos leves y $6 \%$ afirma que es la misma según la época del año.

Un evento poco usual en el pasado son los días calurosos en invierno, como en diciembre, lo cual es percibido como muy frecuente (30\%), ocasionalmente (54\%) e inexistente (16\%). Además, $66 \%$ afirma que hay aumento paulatino de días fríos. El frío viene en una "especie de niebla que quema las plantas y daña la flor de jamaica" (Barrera, comunicación personal, 10 de diciembre de 2018). Para evitar la pérdida cortan la flor tempranamente (tierna). Esta es una de las razones por las que ha disminuido la siembra.

En cuanto a la precipitación, 41 \% estima una reducción drástica del periodo, mientras que para $55 \%$ es moderada. En otras palabras, $96 \%$ de los habitantes se refieren a una disminución del número de días de lluvia durante el temporal que inicia en julio. Este movimiento del "tiempo de siembra" junto con la reducción obligan a utilizar otras semillas, es decir, las mejoradas de ciclo corto en sustitución de las propias (denominadas por los campesinos de maíces nativos o criollos). Sin embargo, el maíz mejorado o híbrido se usa 


\section{Revista Iberoamericana \\ de las Ciencias Sociales y Humanísticas}

ISSN: 2395 - 7972

para la venta, no para el consumo familiar porque no posee las características de olor, sabor y maleabilidad de la masa que tiene el maíz nativo.

Otras modificaciones en las características del temporal son evidentes: en primer lugar, está el menor volumen de agua de lluvia observado por $95 \%$ de los entrevistados, con variantes entre módico (56 \%) y relevante (39\%). Asimismo, se percibe una disminución de lluvia torrencial $(71 \%)$ y un incremento de lluvias asociadas con fuertes vientos y granizo (69 \%): “Antes llovía más por la noche y después de mediodía... Era una lluvia lenta y durante varias horas que penetraba en la tierra... El río tenía agua todo el año... había ciénegas" (García, comunicación personal, 20 de julio de 2019). Ahora hay menos agua y “como no llueve mucho se calienta la tierra", el río se seca, las ciénegas desaparecieron, la "lluvia se prolonga hasta octubre y noviembre, pero pudre la flor de jamaica", con la consecuente pérdida o disminución de la cosecha según los daños. O cae lluvia muy fuerte, "un golpe de agua que se escurre", se pierde, no se absorbe en el suelo, es solo "un pasón" (Barrera, comunicación personal, 10 de diciembre de 2018) que causa daños en vez de beneficios por sequía o porque es erosiva. Además, "por la escasez de agua en el río ya no hay cultivos de riego" (García, comunicación personal, 20 de julio de 2019), los cuales dependen únicamente del ciclo de temporal para la producción del maíz.

La sequía es la manifestación del cambio que más daña el cultivo de maíz. Se entiende por sequía la falta de agua de lluvia por 15 días durante el temporal. Dependiendo de los días de ausencia de lluvia, el jilote o elote no se desarrolla adecuadamente o, en definitiva, se siniestra la cosecha. $68 \%$ de los habitantes considera que padecen sequía frecuente, mientras que $30 \%$ sostiene que es relativa. Incluso algunos afirman que desde hace como 15 años se presenta "un año bueno y uno malo" (Meneses, comunicación personal, 20 de julio de 2019), es decir, las condiciones ya no son adecuadas para la agricultura tradicional y de autoconsumo.

Por su parte, los fuertes vientos que $\operatorname{acaman}^{3}$ la planta de maíz son cada vez más frecuentes para $26 \%$; en cambio, $66 \%$ estima regularidad en su ocurrencia. Si las áreas de acame son amplias o dependiendo de la etapa de desarrollo en que se encuentra la planta de maíz, las pérdidas son importantes. Se dice, por ejemplo, que si se presenta el acame, "la tira

\footnotetext{
${ }^{3}$ Acame es cuando fuertes vientos voltean hasta el suelo las plantas de maíz; puede solo caer o arrancarse desde la raíz, con la consecuente pérdida del fruto. El viento "las tumba", dicen los pobladores.
} 


\section{Revista Iberoamericana \\ de las Ciencias Sociales y Humanísticas}

ISSN: 2395 - 7972

antes de jilotear no habrá elote, se espanta, ya no sale el jilote, ya no da" (Victoriano, comunicación personal, 31 de octubre de 2018). Estos campesinos han encontrado que el maíz mejorado, además de ser de ciclo más corto, tiene mayor resistencia a la sequía y acame, así que son una buena opción para la producción.

En el ciclo de cultivo se observan cuatro etapas claramente diferenciadas: siembra, cosecha, almacenamiento y descanso del suelo. Los cambios en las condiciones climáticas tienen efectos en cada una de ellas y consecuencias directas en la producción de alimentos de esta población.

Un aspecto observado durante las visitas es el abandono del uso de la troje. La troje en este lugar no es una estructura, sino una forma de almacenamiento de la mazorca con su hoja como cubierta de protección. Las mazorcas se colocan por hileras en algún espacio en el interior de la vivienda, y para controlar la presencia de plagas se les dispersa cal. Este desuso - sugieren los campesinos — se debe a que en la actualidad los daños provocados por el gorgojo y otros insectos son cada vez mayores. Así, esta práctica está siendo desplazada por el almacenamiento del maíz en grano en depósitos metálicos o plastificados a los que se adiciona fosfuro de aluminio como insecticida, conocido como "la pastilla". También se observó el desuso de otras prácticas agrícolas como el barbecho; sin embargo, no fue posible determinar si esto está relacionado con aspectos climáticos o no.

Otros indicios reconocidos por los campesinos son la disminución de la vegetación común o nativa en $49 \%$; de hecho, $26 \%$ distingue nuevas plantas en sus terrenos que les son extrañas. "Antes había anís silvestre, ahora ya no se ve" (García, comunicación personal, 20 de julio de 2019). El indicador más sentido entre los pobladores es la limitada disponibilidad de agua de uso doméstico; esto se asume como una afectación contundente para 50 \% y con regularidad para $46 \%$. En otras palabras, $96 \%$ lo reconoce como problema.

Pocos indicadores predictores del clima permanecen vivos o no se han indagado con suficiencia en esta región. Al respecto, se encontró que "cuando el ambiente se presenta brumoso, es señal de sequía y presencia de plagas" durante el temporal. Por brumoso se refieren a días con presencia de densa neblina (Chino, comunicación personal, 31 de octubre de 2018).

Cabe resaltar el reconocimiento como indebidas de sus propias decisiones y actividades; así lo expresa un informante refiriéndose a la producción de maíz: 


\section{Revista Iberoamericana \\ de las Ciencias Sociales y Humanísticas}

ISSN: $2395-7972$

Ya no se cultiva como antes, pues que ahora sí no nos daba trabajo, no liquideábamos, no nada, así pues, nada más se sembraba y no más se limpiaba, y ahorita se cultiva bien por lo mismo de que, pues, ahora sí hay que abonar, hay que ponerle líquido al monte y a las plagas (Barrera, comunicación personal, 10 de diciembre de 2018).

Desde su autovaloración, reconocen lo negativo de sus actuaciones: "La gente se desvió mucho con el uso de los líquidos" (Victoriano, comunicación personal, 31 de octubre de 2018). Incluso utilizan el término liquidiar, entendido como dispersar agroquímicos para secar la hierba o el "monte" (como llaman a las arvenses en los terrenos de cultivo, matalarvas e insectos plaga), pero al mismo tiempo son contaminantes y destructivos de insectos polinizadores. Sin embargo, al no disponer de otras opciones, no usarlos implica pérdidas considerables: "Un cultivo de una hectárea con plaga se destruye en dos o tres días por completo, por eso la gente se desconsuela" (Victoriano, comunicación personal, 31 de octubre de 2018), "ya no se da como antes", "la vida ya no es como antes" (Ayodoro, comunicación personal, 12 de enero de 2019). Así, los agroquímicos han logrado posicionarse como un recurso estratégico hasta para estas poblaciones. Esto, sumado a la deforestación que afecta la precipitación e intensifica el calor, son elementos que en conjunto se convierten en un problema de compleja solución.

\section{Discusión}

En Huamuchapa dos asuntos son de relevancia: sus conceptualizaciones, manejo e implicaciones del tiempo (estrechamente vinculados al cultivo del maíz) y la percepción de indicadores climáticos (algunos poco documentados).

En esta sociedad, el cambio climático, como tal, aún no es nombrado ni dotado de contenido propio. De hecho, quizás ni se requiere porque incluso en las comunidades científicas del mundo no hay una postura única.

No obstante, para los campesinos existe el tiempo. Tiempo es una categoría central, compleja y de larga data, polisémica. Además, es polimorfa o polivalente, y denota periodos y magnitudes uniformes, lineales y medibles en unidades numéricas, pero también ciclos, condiciones, temporalidades y estados cualitativamente distintos, irreversibles e irrepetibles, como los ambientales, atmosféricos y productivos, con un antes y un después. Una de estas formas del tiempo es el clima, el cual algunas veces tiene el mismo sentido y en otras 


\section{Revista Iberoamericana de las Ciencias Sociales y Humanísticas}

ISSN: $2395-7972$

mantiene un vínculo indisoluble. El "tiempo de secas" y el "tiempo de lluvias" son dos condiciones constituyentes de un calendario estacional anual individual y al mismo tiempo colectivo, porque determina una organización específica de la vida social; en ellos se ajustan y desarrollan actividades concretas relacionadas con el ciclo agrícola del maíz. Hasta las decisiones de salida y retorno como jornaleros agrícolas coinciden con estos tiempos.

El clima se asume cambiante, con movimiento y vida propia; de repente parece que nada puede hacerse contra el orden "natural o divino", "un año bueno y uno malo"; sin embargo, su hacer es constante: lo enfrentan, se anticipan, idean, planean, actúan. En un acto de compromiso consigo mismo, con sus familias y con sus creencias.

Sobre la percepción de indicadores, algunos de nuestros hallazgos son coincidentes con otros estudios locales, aunque vale acotar que sobre vegetación existen escasas publicaciones. Por ejemplo, se sabe que las plantas necesitan condiciones óptimas para prosperar y que los cambios ambientales vinculados pueden contribuir a la desaparición o aparición de especies en ciertas áreas. En tal sentido, casi la mitad de estos campesinos, por su amplio conocimiento de la vegetación en su entorno, refieran la pérdida de plantas, y tres de cada diez señalan la presencia de plantas nunca antes vistas.

Es posible que las lluvias asociadas a fuertes vientos y la reducción del periodo ocasionen la disminución de la población de plantas silvestres y la aparición de nuevas especies en los terrenos. Estos elementos en su conjunto son indicios para realizar estudios especializados que lleven a determinar el tipo de vegetación más conveniente, de utilidad comercial, alimenticia u otros para la zona, o para determinar las áreas geográficas convenientes para el mantenimiento de las especies de interés comunitario actual (como el maíz), puesto que es un recurso vital y su proceso de adaptación ha sido largo. Es pertinente experimentar cuál maíz nativo es más resistente a estas circunstancias cambiantes.

Es evidente el reconocimiento de que el clima está cambiando. Ya no se sabe cómo vendrá el temporal. Hace más calor y las modificaciones en el patrón de lluvias retrasan su inicio, que afecta las prácticas tradicionales de cultivo. Estas percepciones son coincidente con varios autores (Barrasa; 2017, Soares et al., 2018; Vélez-Torres et al., 2016). Sin embargo, en nuestro caso, aunque se trata de percepciones campesinas, es posible identificar niveles de detalle y efectos que podrían compararse con datos de las estaciones meteorológicas para una interpretación más completa. 


\section{Revista Iberoamericana de las Ciencias Sociales y Humanísticas}

ISSN: $2395-7972$

Autores como Soares et al. (2018) enfatizan que independiente de la construcción del concepto de cambio climático, a nivel local se observa, interpreta y siente el impacto de las variaciones en los periodos de lluvia y el incremento del calor (Vélez-Torres et al., 2016). En este caso, 8 de cada 10 campesinos consideran que los cambios de clima han sido más notables en los últimos 5 años, aunque hay quienes reconocen que esto ocurre desde hace 10 años y hasta 30 atrás. Esta discrepancia en la temporalidad puede ser debido a la edad de los informantes y a sus condiciones socioeconómicas relacionadas con la pluriactividad que desempeñan.

Por otra parte, quizás la falta de terrenos de cultivo o el disponer cerca de la vivienda de una fuente de suministro de agua para consumo doméstico sean las razones por las cuales $14 \%$ consideró que no hay tal modificación del clima. Ciertamente, hay personas que ven los cambios de clima como un acontecimiento natural, mientras que a otras, aun sabiendo que se trata de eventos que causan daños, les parecen distantes debido a que no se relacionan directamente con sus actividades o bienes, como lo explican los estudios sobre la percepción urbana del cambio climático (Martínez, 2001; Novión y Estrada, 2011; Urbina, 2004).

Para los campesinos de Huamuchapa estos cambios son de suma importancia para la sobrevivencia de la comunidad, de la familia y de las próximas generaciones, por lo que se trata de una población vulnerable. En este sentido, un estudio entre campesinos en Santander refiere consecuencias en los ecosistemas y en las generaciones futuras, aunque pueden solucionarse mediante la acción colectiva (Pinilla et al., 2012). Prácticas tradicionales que no generaban impacto ambiental casi se han perdido (p. ej., el almacenamiento en troje, actividad que se transmitía generacionalmente). El fosfuro de aluminio para conservar granos tiene graves consecuencias en la salud y hasta en la vida de las personas, si no se usa adecuadamente.

En encuestas realizadas en países como Estados Unidos, Malta y Canadá se encontró que las personas relacionan el cambio climático con las altas temperaturas y con efectos en la salud (Leiserowitz, Akerlof, De Bono, Vincenti y Calleja, citados por Salazar-Ceballos et al., 2016).

En el caso de los campesinos de Huamuchapa, explican que estos cambios se deben principalmente a la tala de árboles, la quema de basura, la contaminación, entre otras, lo cual se asemeja con lo reportado por González et al. (2017), aunque vale señalar que en la presente 


\section{Revista Iberoamericana}

de las Ciencias Sociales y

Humanísticas

ISSN: $2395-7972$

investigación también se hallaron explicaciones divinas para justificar dicho fenómeno ambiental.

\section{Conclusiones}

Aunque palabras como tiempo y clima suelen ser usadas con el mismo significado, en Huamuchapa, municipio de Tecoanapa, Guerrero, los campesinos tienen una idea muy clara de lo que está ocurriendo con el clima y de sus efectos en la producción agrícola y en las condiciones ambientales de su comunidad. En tal sentido, consideran que los eventos de mayor relevancia tienen que ver con el incremento en la temperatura (94\%), los cambios en la precipitación (96\%) y la falta de disponibilidad de agua de uso doméstico (96\%), todo lo cual genera incertidumbre en los habitantes. De hecho, durante el temporal han observado la reducción en la cantidad de agua de lluvia, la disminución de los días del periodo, el aumento de lluvias torrenciales, la presencia de vientos fuertes y sequias, así como los cambios en la vegetación.

Esta situación, según la percepción de más de la mitad de estos campesinos, tiene origen antropogénico debido principalmente a la deforestación y a la contaminación, y en menor medida al calentamiento global del planeta.

El impacto de esto se traduce en disminución drástica de las cosechas y, en particular, del maíz casi cada dos años, de ahí que el riesgo de estas familias se incremente, puesto que dicho producto es estratégico para la alimentación. Por eso, y para atenuar los efectos por las modificaciones en la precipitación, sequía y acame por el viento, están reemplazando los maíces nativos por híbridos, lo cual si bien no resulta beneficioso para la conservación de la biodiversidad del maíz nativo, constituye la única vía para garantizar la sobrevivencia.

Por otra parte, también fue posible evidenciar que la valoración que dan a los eventos climáticos depende de si tienen o no consecuencias directas en sus vidas. Por ello, para algunos los cambios climáticos no representan problema alguno, por lo que no modifican sus actuaciones; en cambio, los campesinos que se dedican al cultivo de maíz y otros productos lamentan profundamente lo que está ocurriendo.

Asimismo, como los cambios climáticos se están generando vertiginosamente y los procesos adaptativos son más lentos, se requiere de tiempo (un ciclo agrícola completo) para probar y otros más para afianzar un resultado. Así, el reconocimiento de que "el tiempo ha cambiado mucho" implica añoranza de un pasado sin retorno del que se tiene plena 


\section{Revista Iberoamericana \\ de las Ciencias Sociales y Humanísticas}

ISSN: 2395 - 7972

conciencia. Al respecto, algunos reconocen que su propio actuar incide en la naturaleza y que si hoy no revierte esta realidad, será más drástico para las generaciones futuras.

Este tipo de acercamiento a nivel local identificó las pérdidas asociadas al clima de impacto cultural (como la troje) y de elementos predictores climáticos (como las cabañuelas), así como el reconocimiento de cambios en la vegetación que alteran la diversidad biológica. Asimismo, mostró que el elemento más significativo en riesgo es el maíz (en particular el nativo), producto humano que no puede existir sin la intervención de hombres o mujeres, por lo que en él se sintetiza el ambiente, la cultura y la diversidad biológica.

Por otro lado, el estudio demostró pertinencia en la identificación de cambios de clima y resiliencia en estos campesinos, quienes emprenden acciones con recursos propios para enfrentar las eventualidades climáticas. Es la necesidad de disponer de alimentos lo que lleva a un hacer constante, aunque al mismo tiempo muestra contradicciones, puesto que ese proceder involucra el uso de procedimientos y productos que resuelven un problema de alimentación en lo inmediato, pero también contribuye a su inviabilidad a largo plazo.

No podemos concluir sin dejar de mencionar algunas potencialidades y limitaciones para este tipo de trabajos. Sin duda, las posibilidades de investigación son amplias, las cuales se pueden enfocar en proponer energías sustentables para disminuir la tala y la contaminación. Asimismo, indagar sobre el conocimiento de las mujeres en torno a los cambios de clima, lo cual puede abarcar desde cómo los nombran hasta las acciones que toman para afrontarlos. Igualmente, profundizar en el conocimiento de estrategias adaptativas, realizar estudios de intervención para desarrollar prácticas y comportamientos amigables con el medio ambiente, así como encontrar variedades de maíz más adaptables a estas condiciones cambiantes, pero que preserven su riqueza biocultural, la cual ha sido generada durante siglos de trabajo humano.

Este tipo de estudios pueden realizarse no solo en esta región, sino en cualquiera otra de la entidad, pues en Guerrero son constantes los eventos climáticos catastróficos. De lograrse un verdadero diálogo de saberes entre campesinos, científicos y autoridades, las repuestas pueden ser realmente positivas.

Finalmente, en cuanto a las limitaciones, se pueden mencionar la falta de recursos para el trabajo de campo y la elección de los contactos pertinentes para la apertura comunitaria. Lo más relevante durante el levantamiento de la encuesta fue desconocer el primer día, que en la comunidad había horarios de apertura y cierre de acceso establecidos 
Revista Iberoamericana

de las Ciencias Sociales y

Humanísticas

ISSN: 2395 - 7972

por el grupo de autodefensa. El desconocimiento de la dinámica comunitaria puede ser una limitante importante. No obstante, ninguno de estos aspectos fue imposible de resolver, y fue compensado con la accesibilidad y la confianza de las personas participantes.

\section{Referencias}

Agencia Europea de Medio Ambiente [AEMA]. (2006). Conjunto básico de indicadores de la AEMA, Guía. Ministerio de Medio Ambiente. Recuperado de https://www.miteco.gob.es/es/calidad-

yevaluacionambiental/publicaciones/ConjuntoBasicoIndicadores_tcm30-185692.pdf

Ametrano, L., Vestfrid P., Bonaparte, A., Adaro, R., Huarte, F. (2017). Técnicas de Investigación Social. Universidad Nacional de la Plata. Facultad de Periodismo y Comunicación Social, pág. 143.

Bárcena, A., Samaniego, J. L., Peres, W. y Alatorre, J.E. (2020). La emergencia del cambio climático en América Latina y el Caribe: ¿seguimos esperando la catástrofe o pasamos a la acción? Comisión Económica para América Latina y el Caribe (Cepal), n. ${ }^{\circ}$ 160. Santiago, Chile.

Barrasa, S. (2017). Percepción del cambio climático en comunidades campesinas de la Reserva de la Biósfera La Encrucijada, Chiapas, México. Cuadernos Geográficos de la Universidad de Granada, 56(3), 44-65.

Cea, D. M. A. (2001). Metodología Cuantitativa. Estrategias y técnicas de investigación social. Editorial Síntesis. España, pág. 415.

Corona, M. A. (2018). El conocimiento, la percepción y disponibilidad para afrontar el cambio climático en una población emergente, los migrantes de retorno. Estudios Sociales, 28(52), 1-17. Recuperado de https://www.ciad.mx/estudiosociales/index.php/es/article/view/578/360

Detta, E. (2014). Las tres verdades del cambio climático que ya deberías conocer. Banco Interamericano de Desarrollo [BID]. Recuperado de https://blogs.iadb.org/sostenibilidad/es/cosas3saberdeberia/

Edwards, G., García, A. y Walkins, G. (25 de marzo de 2020) ¿Cuál es el vínculo entre covid19 y las emergencias ecológicas y climáticas? Banco Interamericano de Desarrollo [BID]. Recuperado de https://blogs.iadb.org/sostenibilidad/es/cual-es-el-vinculoentre-covid-19-y-las-emergencias-ecologicas-y-climaticas/ 
Revista Iberoamericana

de las Ciencias Sociales y

Humanísticas

ISSN: 2395 - 7972

Estrada, P. F. (2017). El calentamiento local en ciudades, mayor al calentamiento global. Boletín UNAM-DGCS-391. Recuperado de http://www.dgcs.unam.mx/boletin/bdboletin/2017_391.html

Ferrero, E. L., Hernández, Y. T. y Zafra, C. F. (2014). Percepción latinoamericana de cambio climático: metodologías, herramientas y estrategias de adaptación en comunidades locales. Una revisión. U.D.C.A Actualidad \& Divulgación Científica, 17(1), 73-85.

Flick, U. (2007). En Introducción a la Investigación Cualitativa, Ediciones Morata S. L. Madrid, pág. 313.

González, S. Ll., Silva, J. T., Ávila, L. A., Moncayo-Estrada, R., Cruz, G. y Ceja, L. F. (2017). El fenómeno de cambio climático en la percepción de la comunidad indígena purépecha del municipio de Chilchota, Michoacán, México. Revista Internacional de Contaminación Ambiental, 33(4), 641-653. Doi: 10.20937/RICA.2017.33.04.08

Hernández, S. R., Fernández, C. C., y Baptista, L. P (2003). Metodología de la Investigación. Mc Graw Hill. México, pág. 705.

Mariño, N. (2011). Reflexiones sobre la perspectiva cultural en las políticas de cambio climático en Colombia: un acercamiento al análisis cultural y espacial de las políticas públicas. En Ulloa, A. (ed.), Perspectivas culturales del clima (pp. 495-527). Bogotá, Colombia: Universidad Nacional de Colombia, ILSA.

Martínez, C. A. (2015). Percepción pública del cambio climático en México (tesis de maestría). Universidad Iberoamericana, México. Recuperado de http://ri.ibero.mx/handle/ibero/698

Munguía-Aldama, J., Sánchez-Plata, F., Vizcarra-Bordi, I. y Rivas-Guevara, M. (2015). Estrategias para la producción de maíz frente a los impactos del cambio climático. Revista de Ciencias Sociales, 21(4), 538-547.

Novión, A. C. y Estrada, G.C. (2011). Percepción de los efectos vivenciales del cambio climático en una muestra de habitantes urbanos australes. Magallania, 39(1), 93-102.

Pinilla, M. C., Rueda, A., Pinzón, A. y Sánchez, J. (2012). Percepciones sobre los fenómenos de variabilidad climática y cambio climático entre campesinos del centro de Santander, Colombia. Ambiente y Desarrollo, 16 (31), 25-37.

Salazar-Ceballos, A., Freyle, N., Tamara, G. y Álvarez-Miño, L. (2016). Percepción sobre riesgo al cambio climático como una amenaza para la salud humana, Taranga, Santa Marta, 2014. Luna Azul, (43), 102-127. Doi: 10.17151/luaz.2016.43.6 
Soares, D., García, A. y Manzano, L. R. (2018). Cambio climático. Percepciones sobre manifestaciones, causas e impactos en el Distrito de Temporal Tecnificado Margaritas-Comitán, Chiapas. Ciencia Ergo Sum, 25(1). Recuperado de http://www.redalyc.org/articulo.oa?id=10453975001

Tamayo y Tamayo, M. (2002). El proceso de la investigación científica. Limusa. México, pág. 440.

Taylor, S.J. y Bogdan, R (2013). En Introducción a los métodos cualitativos de investigación. Paidós. España, pág. 343.

Urbina, J. (2015). La percepción social del cambio climático en el ámbito urbano. En Ortiz Espejel, B. y Velazco Samperio, C. (coords.), La percepción social del cambio climático. Estudios y orientaciones para la educación ambiental en México (pp. 2236). Universidad Iberoamericana de Puebla. Recuperado de http://repositorio.iberopuebla.mx

Ulloa, A. (2011). Construcciones culturales sobre el clima. En Ulloa, A. (ed.), Perspectivas culturales del clima (pp. 33-53). Bogotá, Colombia: Universidad Nacional de Colombia, ILSA.

Vélez-Torres, A., Santos-Ocampo, A., De la Tejera-Hernández, B. G. y Monterroso-Rivas, I. A. (2016). Percepción del cambio climático en los agricultores periurbanos y rurales del municipio de León, Guanajuato. Geografía Agrícola, (57), 7-18. Doi: 10.5154/r.rga.2016.57.008 


\section{Revista Iberoamericana \\ de las Ciencias Sociales y Humanísticas}

\begin{tabular}{|c|c|}
\hline $\begin{array}{l}\text { Rol de } \\
\text { Contribución }\end{array}$ & Autor (es) \\
\hline Conceptualización & $\begin{array}{l}\text { Josefina Munguia-Aldama (Grado de contribución principal) } \\
\text { Eleuterio Campos-Hernández (Grado de contribución principal) } \\
\text { Rosalba Díaz-Vásquez (Grado de contribución igual) } \\
\text { Mario Martínez-Rescalvo (Grado de contribución igual) } \\
\text { Roxana Reyes-Ríos (Grado de contribución igual) }\end{array}$ \\
\hline Metodología & $\begin{array}{l}\text { Josefina Munguia-Aldama (Grado de contribución principal) } \\
\text { Eleuterio Campos-Hernández (Grado de contribución principal) } \\
\text { Rosalba Díaz-Vásquez (Grado de contribución igual) } \\
\text { Mario Martínez-Rescalvo (Grado de contribución igual) } \\
\text { Roxana Reyes-Ríos (Grado de contribución igual) }\end{array}$ \\
\hline Validación & $\begin{array}{l}\text { Josefina Munguia-Aldama (Grado de contribución principal) } \\
\text { Eleuterio Campos-Hernández (Grado de contribución principal) } \\
\text { Rosalba Díaz-Vásquez (Grado de contribución igual) } \\
\text { Mario Martínez-Rescalvo (Grado de contribución igual) } \\
\text { Roxana Reyes-Ríos (Grado de contribución igual) }\end{array}$ \\
\hline Análisis Formal & $\begin{array}{l}\text { Josefina Munguia-Aldama (Grado de contribución principal) } \\
\text { Eleuterio Campos-Hernández (Grado de contribución principal) } \\
\text { Rosalba Díaz-Vásquez (Grado de contribución igual) } \\
\text { Mario Martínez-Rescalvo (Grado de contribución igual) } \\
\text { Roxana Reyes-Ríos (Grado de contribución igual) }\end{array}$ \\
\hline Investigación & $\begin{array}{l}\text { Josefina Munguia-Aldama (Grado de contribución principal) } \\
\text { Eleuterio Campos-Hernández (Grado de contribución principal) } \\
\text { Rosalba Díaz-Vásquez (Grado de contribución igual) } \\
\text { Mario Martínez-Rescalvo (Grado de contribución igual) } \\
\text { Roxana Reyes-Ríos (Grado de contribución igual) }\end{array}$ \\
\hline Curación de datos & $\begin{array}{l}\text { Josefina Munguia-Aldama (Grado de contribución principal) } \\
\text { Eleuterio Campos-Hernández (Grado de contribución principal) } \\
\text { Rosalba Díaz-Vásquez (Grado de contribución igual) } \\
\text { Mario Martínez-Rescalvo (Grado de contribución igual) } \\
\text { Roxana Reyes-Ríos (Grado de contribución igual) }\end{array}$ \\
\hline $\begin{array}{l}\text { Escritura - } \\
\text { Preparación del } \\
\text { borrador original }\end{array}$ & $\begin{array}{l}\text { Josefina Munguia-Aldama (Grado de contribución principal) } \\
\text { Eleuterio Campos-Hernández (Grado de contribución principal) } \\
\text { Rosalba Díaz-Vásquez (Grado de contribución igual) } \\
\text { Mario Martínez-Rescalvo (Grado de contribución igual) } \\
\text { Roxana Reyes-Ríos (Grado de contribución igual) }\end{array}$ \\
\hline $\begin{array}{l}\text { Escritura - Revisión } \\
\text { y edición }\end{array}$ & $\begin{array}{l}\text { Josefina Munguia-Aldama (Grado de contribución principal) } \\
\text { Eleuterio Campos-Hernández (Grado de contribución principal) } \\
\text { Rosalba Díaz-Vásquez (Grado de contribución igual) }\end{array}$ \\
\hline
\end{tabular}


Revista Iberoamericana de las Ciencias Sociales y Humanísticas

Mario Martínez-Rescalvo (Grado de contribución igual)

Roxana Reyes-Ríos (Grado de contribución igual) 